\title{
Nearest-Neighbor Correlations in Hubbard Model
}

\author{
Yu.B.Kudasov* \\ Russian Federal Nuclear Center - VNIIEF \\ pr. Myra 37, Sarov, Nizhni Novgorod \\ Region, Russia, 607190
}

(April 26, 2018)

The Hubbard Hamiltonian is investigated by means of a new variational trial wave function. The trial wave function includes either intrasite and nearest - neighbor correlations in an explicit form. To calculate density matrices the method of Kikuchi's pseudoensemble is used. The case of half-filled fermionic band is carefully investigated in the limit of a large number of lattice sites. The ground state energy and correlation functions are determined for Bethe lattices with $z=2,4$ and 6 nearest neighbors.

PACS: 71.10.Fd, 71.10.Hf, 71.27.+a, 71.28+d

Key words: strongly correlated fermions, trial wave function, nearest neighbor correlations

*E-mail: kudasov@ntc1.vniief.ru 
Short-range correlations have been shown to have a considerable influence on properties of strongly interacting fermions. In the most refined way, the problem is stated within the framework of the one-band Hubbard model [1] 3 . If fermions of spin $1 / 2$ hop to the adjacent sites of a lattice only, the Hubbard Hamiltonian has the following form

$$
H=H_{k}+H_{p}=t \sum_{\langle i j\rangle, \sigma}\left(a_{i \sigma}^{\dagger} a_{j \sigma}+H . c .\right)+U \sum_{i} n_{i \uparrow} n_{i \downarrow}
$$

where $a_{i \sigma}^{\dagger}\left(a_{j \sigma}\right)$ is the creation (annihilation) operator of a fermion of spin $\sigma$ on the $i$-th lattice site, $\langle i j\rangle$ denotes a pair of adjacent sites, $n_{i \sigma}=a_{i \sigma}^{\dagger} a_{j \sigma}$.

There are known few exact solutions of the Hamiltonian (11): for a homogeneous chain (1D) [4, so-called Gutzwiller approach (GA) for a lattice of infinitive dimensions $\mathrm{D}=\infty$ [2,5] and some other special cases [6,7]. Lattices of intermediate dimensions, especially $2 \mathrm{D}$ and $3 \mathrm{D}$ ones, are of great practical importance, and a number of works is devoted to their investigation (see [8,9] and reference therein). In particular, the Gutzwiller's trial wave function which is exact in the limit of $\mathrm{D}=\infty$ was applied [10 [12]. This wave function entered in a numerical procedure of the variational Monte Carlo (VMC) method [10] or was used in diagrammatic expansions of GA in terms of 1/D [11,12]. In the last case, one had to base on a strong assumption that the ground state of 2D and 3D lattices only slightly differed from that of the $\mathrm{D}=\infty$ lattice. Actually, the problem of lattices of finite dimensions lies in necessity of inclusion of spacial correlations, especially short-range ones. As was shown phenomenologically in the framework of Nearly Antiferromagnetic Fermi Liquid theory [13], in some situations the short-range correlations are very strong and, therefore, a microscopic non-perturbative approach is needed to treat them.

In the present Letter, we explore the Hamiltonian (1) with half-filled initial fermionic band by means of a trial wave function of Gutzwiller's type which, in addition to intrasite correlations, includes nearest-neighbor ones in an explicit form. Let us consider a system of $N$ fermions of spin $1 / 2$ on a lattice consisted of $L$ sites. Then, in a general form, the correlated $N$-particle trial wave function can be written as 


$$
|\psi\rangle=\prod_{\lambda} g_{\lambda}^{\widehat{P}_{\lambda}}\left|\varphi_{0}\right\rangle
$$

where $\left|\varphi_{0}\right\rangle$ is the $N$-particle wave function of free fermions, for instance the Fermi sea $\prod_{k<k_{F \uparrow}} a_{k \uparrow}^{\dagger} \prod_{k<k_{F \downarrow}} a_{k \downarrow}^{\dagger}|0\rangle, k$ is the wave number of a fermion, $k_{F \sigma}$ is the Fermi wave number of fermions of spin $\sigma, g_{\lambda}$ are the parameters which lies within interval $] 0, \infty\left[, \widehat{P}_{\lambda}\right.$ are the projection operators onto all feasible configurations of a lattice site and a pair of adjacent sites. There are 4 such operators for itrasite configurations

$$
\widehat{X}_{1}=\sum_{i}\left(1-n_{i \uparrow}\right)\left(1-n_{i \downarrow}\right), \widehat{X}_{2}=\sum_{i} n_{i \uparrow}\left(1-n_{i \downarrow}\right), \widehat{X}_{3}=\sum_{i}\left(1-n_{i \uparrow}\right) n_{i \downarrow}, \widehat{X}_{4}=\sum_{i} n_{i \uparrow} n_{i \downarrow}
$$

and 10 operators for nearest neighbor configurations,

$$
\widehat{Y}_{1}=\sum_{\langle i j\rangle}\left(1-n_{i \uparrow}\right)\left(1-n_{i \downarrow}\right)\left(1-n_{j \uparrow}\right)\left(1-n_{j \downarrow}\right), \widehat{Y}_{2}=\sum_{\langle i j\rangle} n_{i \uparrow} n_{i \downarrow} n_{j \uparrow} n_{j \downarrow}, \text { and etc. }
$$

(see Table 【).

The trial wave function (2) remains to be antisymmetric and conserves translational properties of $\left|\varphi_{0}\right\rangle$. On the other hand, either intrasite and nearest-neighbor correlations are taken into consideration explicitly. From now on, we shall consider lattices for which the total number of nearest-neighbors pairs is equal to $z L / 2$, where $z$ is the number of nearest neighbors of a site. Let us denote normalized eigenvalues of the projection operators as $x_{\lambda}|\Phi\rangle=L^{-1} \widehat{X}_{\lambda}|\Phi\rangle, y_{\lambda}|\Phi\rangle=(z L / 2)^{-1} \widehat{Y}_{\lambda}|\Phi\rangle$. The eigenvalues turn out to be related to each other by normalization conditions [14]

$$
\sum_{\lambda} x_{\lambda}=1, \sum_{\lambda} \beta_{\lambda} y_{\lambda}=1
$$

and self-consistency conditions [14]

$$
\begin{aligned}
& y_{1}+y_{3}+y_{4}+y_{5}=x_{1}, \\
& y_{2}+y_{3}+y_{8}+y_{9}=x_{4}, \\
& y_{4}+y_{6}+y_{7}+y_{8}=x_{2}, \\
& y_{5}+y_{7}+y_{9}+y_{10}=x_{3} .
\end{aligned}
$$


As concentrations of fermions of each spins are fixed there are the only independent parameter $x_{\lambda}$ and 7 independent parameters $y_{\lambda}$. In the case of half-filled band, additional constrains appear

$$
y_{1}=y_{2}, y_{6}=y_{10}, y_{4}=y_{5}=y_{8}=y_{9}
$$

which reduce the number of independent parameters $y_{\lambda}$ to 3 . It should be noted that, in contrast to (5) and (6), the condition (7) imposes the restriction on averaged values of $y_{i}$ only, that is, configurations, which violate (7), enter into the trial wave function (2). Nevertheless, as will be shown below, one can omit them in the limit $L \longrightarrow \infty$.

Assume that $x_{1}=x_{4}=x, y_{3}, y_{4}$, and $y_{7}$ are independent parameters. Then, taking into account additional degeneracy of the half-filled band, the correlated trial wave function is transformed to

$$
|\psi\rangle=g_{0}^{\widehat{X}} g_{3}^{\beta_{3} \widehat{Y}_{3}} g_{4}^{4 \beta_{4} \widehat{Y}_{4}} g_{7}^{\beta_{7} \widehat{Y}_{7}}\left|\varphi_{0}\right\rangle
$$

At first, we calculate the norm of the correlated trial wave function

$$
\langle\psi \mid \psi\rangle=\sum_{\left\{x, y_{3}, y_{4}, y_{7}\right\}} W_{\left\{x, y_{3}, y_{4}, y_{7}\right\}} g_{0}^{2 L x} g_{3}^{2 z L y_{3}} g_{4}^{8 z L y_{4}} g_{7}^{2 z L y_{7}}=\sum_{\left\{x, y_{3}, y_{4}, y_{7}\right\}} R_{\left\{x, y_{3}, y_{4}, y_{7}\right\}}
$$

where the set $\left\{x, y_{3}, y_{4}, y_{7}\right\}$ describes configurations which contains $L x$ doubly occupied sites of the lattice, $z L y_{3} / 2$ nearest neighbor pairs of $\widehat{Y}_{3}$ type and etc. A unessential factor is omitted in (9) for simplicity. The summation is extended over all possible sets $\left\{x, y_{3}, y_{4}, y_{7}\right\}$. $W_{\left\{x, y_{3}, y_{4}, y_{7}\right\}}$ is the number of configurations with the fixed set of the independent parameters. To calculate $W_{\left\{x, y_{3}, y_{4}, y_{7}\right\}}$ we shall use the Kikuchi's pseudo-ensemble method 14,15. It should be mentioned that this method is exact on the Bethe lattice and approximated for lattices with closed paths [14]. According to Kikuchi's hypothesis, we have

$$
W=\Gamma Q, Q=\frac{(z L / 2) !}{\prod_{\lambda}\left[\left(z y_{\lambda} L / 2\right) !\right]^{\beta_{i}}}, \Gamma=\frac{L ! \prod_{\lambda}\left(x_{\lambda} z L\right) !}{(z L) ! \prod_{\lambda}\left(x_{\lambda} L\right) !}
$$

where lower indexes of $W, \Gamma$, and $Q$ are omitted for the sake of simplicity. $Q$ determines a number of arrangements of 10 elements corresponding to $\widehat{Y}_{\lambda}$ taken $z L / 2$ at a time. $\Gamma$ 
is a fraction of correct arrangements in the pseudo-ensemble. In Eq.(10), dependent $x_{\lambda}$ and $y_{\lambda}$ should be expressed in terms of $x, y_{3}, y_{4}$, and $y_{7}$. In the usual fashion, in the thermodynamic limit $L \rightarrow \infty$, we retain only the terms of the series (9) which are very close to the largest one. As far as $R_{\left\{x, y_{3}, y_{4}, y_{7}\right\}}$ is a positive function, one can search a maximum of its logarithm instead of the function itself. Let us retain the main terms on $L$ only after taking the logarithm. It can be shown that this approach corresponds to substitution $(z L / 2) ! \longrightarrow(L !)^{z / 2}$ which was usually used [14,15]. Then we obtain

$\frac{\ln W}{L}=2(z-1)\left[x \ln x+\left(\frac{1}{2}-x\right) \ln \left(\frac{1}{2}-x\right)\right]-z\left[y_{2} \ln y_{2}+y_{3} \ln y_{3}+4 y_{4} \ln y_{4}+y_{6} \ln y_{6}+y_{7} \ln y_{7}\right]$

where $y_{2}=x-y_{3}-2 y_{4}, y_{6}=1 / 2-x-y_{7}-2 y_{4}$. The domain of function (11) where we search the maximum is limited by conditions (5) and (6). At its boundaries the gradient of function (11) is directed inwards the domain. That is why, the global maximum of $\ln R / L$ should be an inner one, and conditions $\partial \ln R / \partial \eta_{\lambda}=0$ where $\eta_{\lambda}=x, y_{3}, y_{4}, y_{7}$ are necessary for the global maximum. They lead to the following system of equations which relate $g_{i}$ to $x$ and $y_{i}$

$$
\begin{aligned}
g_{0} & =\left(\frac{1 / 2-x}{x}\right)^{z-1}\left(\frac{x-y_{3}-2 y_{4}}{1 / 2-x-y_{7}-2 y_{4}}\right)^{z / 2}, \\
g_{3}^{2} & =\frac{y_{3}}{x-y_{3}-2 y_{4}}, \\
g_{4}^{4} & =\frac{y_{4}^{2}}{\left(1 / 2-x-y_{7}-2 y_{4}\right)\left(x-y_{3}-2 y_{4}\right)}, \\
g_{7}^{2} & =\frac{y_{7}}{1 / 2-x-y_{7}-2 y_{4}} .
\end{aligned}
$$

To determine the expectation value of the Hamiltonian (1) we need to calculate a density matrix of the first order:

$$
\rho_{1}=\frac{1}{L} \frac{\left\langle\psi\left|\sum_{<i j>, \sigma}\left(a_{i \sigma}^{\dagger} a_{j \sigma}+H . c .\right)\right| \psi\right\rangle}{\langle\psi \mid \psi\rangle} .
$$

In contrast to GA, while a fermion hops from site $i$ to $j$, it should be taken into account that configurations of adjacent pairs $k-i$ and $i-l$ change (Fig.11). Let us fix a particular 
lattice fragment (Fig.11), and calculate function $W^{\prime}$ of the remain lattice from Eqs.(10). Then, a fraction of configurations, which contain the fragment, can be found as

$$
\frac{W^{\prime}}{W}=\frac{y_{(i j)} \prod_{k} y_{(k i)} \prod_{l} y_{(j l)}}{x_{(i)} x_{(j)}} .
$$

Using (14), we sum up all contributions to the density matrix. Then, it takes the following form

$$
\rho_{1}=4\left[2 y_{4}\left(a_{1} a_{2}\right)^{z-1}+\frac{y_{3} g_{7}}{g_{0} g_{3}} a_{1}^{2(z-1)}+\frac{y_{7} g_{0} g_{3}}{g_{7}} a_{2}^{2(z-1)}\right],
$$

where

$$
a_{1} \quad=\quad\left(y_{2} g_{4}+y_{3} g_{4} / g_{3}+y_{4}\left(g_{7}+1\right) / g_{4}\right) / x
$$

and $a_{2}=\left(y_{6} g_{4}+y_{7} g_{4} / g_{7}+y_{4}\left(g_{3}+1\right) / g_{4}\right) /(1 / 2-x)$. The first term of (15) describes a motion of a fermion in the Hubbard subbands. The second and third ones arise from transitions between the subbands. In this expression, parameters $g_{0}, g_{3}, g_{4}, g_{7}$ should be excluded by means of system (12). After some simplifications we find

$$
\rho_{1}=8\left(y_{4}+\sqrt{y_{3} y_{7}}\right)\left[\frac{y_{4}}{x(1 / 2-x)}\left(\sqrt{y_{2}}+\sqrt{y_{3}}+\sqrt{y_{6}}+\sqrt{y_{7}}\right)^{2}\right]^{z-1} .
$$

Finally, the total energy can be presented in the Gutzwiller's form

$$
E=\frac{1}{L} \frac{\langle\psi|H| \psi\rangle}{\langle\psi \mid \psi\rangle}=q \varepsilon_{0}+x U
$$

where $q=\rho_{1} / \rho_{1}^{0}, \rho_{1}^{0}$ is the density matrix (16) at $U=0, \varepsilon_{0}$ is the average energy of the free

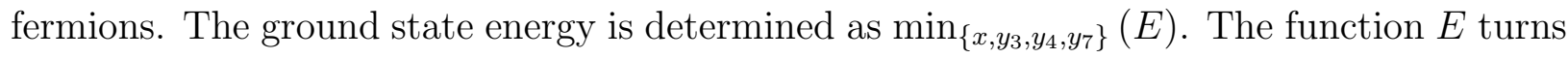
out to be smooth, and its minimum is easily found numerically. It also should be noted that the total energy is invariant in respect of the following substitution: $U \rightarrow-U, y_{7} \longleftrightarrow$ $y_{3}, g_{7} \longleftrightarrow g_{3}, g_{0} \longleftrightarrow 1 / g_{0}$. A detailed discussion of the method presented above will be published elsewhere.

As was mentioned above, the method used is exact for a Bethe lattice. We consider our results for $z=4$ and 6 as approximate solutions for 2D and 3D lattices correspondingly. They are shown in the Fig.22, together with that of 1D chain, and compared with the exact solutions for $1 \mathrm{D}$ chain [4], $\mathrm{D}=\infty$ lattice [2], and numerical results of VMC method for 
a paramagnetic phase [10]. The perturbative expansion $\left(\mathrm{GA}+1 / \mathrm{D}+1 / \mathrm{D}^{2}\right)$ is not shown because it is very close to the GA curve for $2 \mathrm{D}$ and $3 \mathrm{D}$ (a shift of critical values of $U$ between $\mathrm{GA}$ and $\mathrm{GA}+1 / \mathrm{D}+1 / \mathrm{D}^{2}$ is a few percent only 12 ). To investigate the $\mathrm{D}=\infty$ limit of our model we carried out calculations for $z=50,100,200$. They show that the ground state energy tends to the exact $\mathrm{D}=\infty$ solution while $z$ (or $\mathrm{D}$ ) increasing. As one can see from the Fig.2, at intermediate interaction of fermions $(U \sim 1)$, our method gives the ground state energy significantly lower than that of $\mathrm{VMC}$ or $\mathrm{GA}+1 / \mathrm{D}+1 / \mathrm{D}^{2}$ procedures.. This means that nearest-neighbor correlation are essential in this region.

Symmetric and antisymmetric correlation functions of the nearest neighbor

$$
\begin{aligned}
& G_{s}=\left\langle n_{\uparrow} n_{\uparrow}\right\rangle^{\prime}+\left\langle n_{\downarrow} n_{\downarrow}\right\rangle^{\prime}=2\left(y_{2}+2 y_{4}+y_{6}\right), \\
& G_{a}=\left\langle n_{\uparrow} n_{\downarrow}\right\rangle^{\prime}+\left\langle n_{\downarrow} n_{\uparrow}\right\rangle^{\prime}=2\left(y_{2}+2 y_{4}+y_{7}\right)
\end{aligned}
$$

are shown in Fig. 3 for the same lattices as in Fig.2. The prime in Eqs.18 denotes the averaging over nearest-neighbor pairs only. One can see in Fig. 3 that even at $U=0$ some correlations appear due to a exchange hole. An increasing of $U$ leads to enhancing of correlations but there exists a saturation point. At further increasing of $U$ the nearestneighbor correlations remain almost constant and variation of the ground state energy is due to intrasite correlations only.

In conclusion, a new non-perturbative approach to problem of strongly correlated fermions is reported. A trial wave function which includes nearest-neighbor correlations is constructed. For a half-filled initial fermionic band, the ground state energy of the Hubbard Hamiltonian as well as correlation functions are calculated for 2D and 3D lattices and the $1 \mathrm{D}$ chain. Since the correlated wave function $|\psi\rangle$ has the same translational properties as $\left|\varphi_{0}\right\rangle$ does, all the results obtained above describe a paramagnetic state. To build entire phase diagram for Hubbard model our approach need to be extended to an antiferromagnetic phase. The method may be especially beneficial when systems with strong short-range correlations, for example $\mathrm{CuO}_{2}$ planes of HSTC, is considered.

The author thanks Prof. J.Brooks and Dr. W.Lewis for their continuous encouragement. 
The work was supported by the International Science and Technology Center under Project \#829.

[1] J. Hubbard, Proc. Roy. Soc. London A 276, (1963) 238

[2] M. C. Gutzwiller, Phys. Rev. 137, (1965) A1726

[3] J. Kanamory, Prog. Theor. Phys. 30, (1963) 275

[4] H. Lieb and F. Wu, Phys. Rev. Lett. 20, (1968) 1445

[5] W. Metzner and D. Vollhardt, Phys. Rev. Lett. 62, (1989) 324

[6] E. B. Kolomeinsky, J. P. Straley, Rev. Mod. Phys. 68 (1996) 175

[7] U. Brandt, A. Giesekus, Phys. Rev. Lett. 68 (1992) 2648

[8] A. Georges, G. Kotliar, W.Krauth, and M.J.Rozenberg, Rev. Mod. Phys. 68 (1996) 13

[9] G. Senatore, and N.H.March, Rev. Mod. Phys. 66 (1994) 445

[10] H. Yokoyama, H. Shiba, Jour. Phys. Soc. Jap. 56, (1987) 1490; 56, (1987) 3582

[11] W. Metzner and D. Vollhardt, Phys. Rev. Lett. 59, (1989) 121

[12] F. Gebhard, Phys. Rev. B 41, (1990) 9452

[13] A. Millis, H. Monien, and D. Pines, Phys. Rev. B 42, (1990) 167; D. Pines and B. Stojkovic, Phys. Rev. B 55, (1997) 8576

[14] J. M. Ziman, Models of Disorder (Cambridge University Press, Cambridge, London, 1979)

[15] R. Kikuchi, Phys. Rev. 81, (1951) 988 
FIG. 1. A fragment of a tree $(z=4)$. While a fermion hops from $j$ to $k$ site, configurations of adjacent pairs need to be taken into account.

FIG. 2. The ground state energy obtain by minimization of Eq.(17) for 1 D chain $(z=2), z=4$ (2D), and $z=6$ (3D) lattices (solid lines); the exact solutions: GA ( dotted line) [2], 1D chain (dash-dot line) [4]; and numerical VMC calculations (dash line).

FIG. 3. The correlation functions $G_{s}$ (dashed lines) and $G_{a}$ (solid lines) of $1 \mathrm{D}$ chain, $z=4(2 \mathrm{D})$ and $z=6(3 \mathrm{D})$ lattices.

TABLE I. Pair projection operators, corresponding configurations and the degeneracy factor

\begin{tabular}{cccc}
\hline \hline Operator & \multicolumn{2}{c}{ Configuration } & Degeneracy \\
$\widehat{Y}_{i}$ & Site A & Site B & $\beta_{i}$ \\
\hline$\widehat{Y}_{1}$ & $\uparrow \downarrow$ & 1 \\
$\widehat{Y}_{2}$ & $\uparrow \downarrow$ & 1 \\
$\widehat{Y}_{3}$ & $\uparrow$ & $\uparrow$ & 2 \\
$\widehat{Y}_{4}$ & $\uparrow$ & & 2 \\
$\widehat{Y}_{5}$ & $\downarrow$ & & 2 \\
$\widehat{Y}_{6}$ & $\uparrow$ & $\uparrow$ & 1 \\
$\widehat{Y}_{7}$ & $\uparrow$ & $\downarrow$ & 2 \\
$\widehat{Y}_{8}$ & $\uparrow \downarrow$ & 2 \\
$\widehat{Y}_{9}$ & $\uparrow$ & $\uparrow$ & 2 \\
$\widehat{Y}_{10}$ & $\downarrow$ & $\downarrow$ & 1 \\
\hline \hline
\end{tabular}




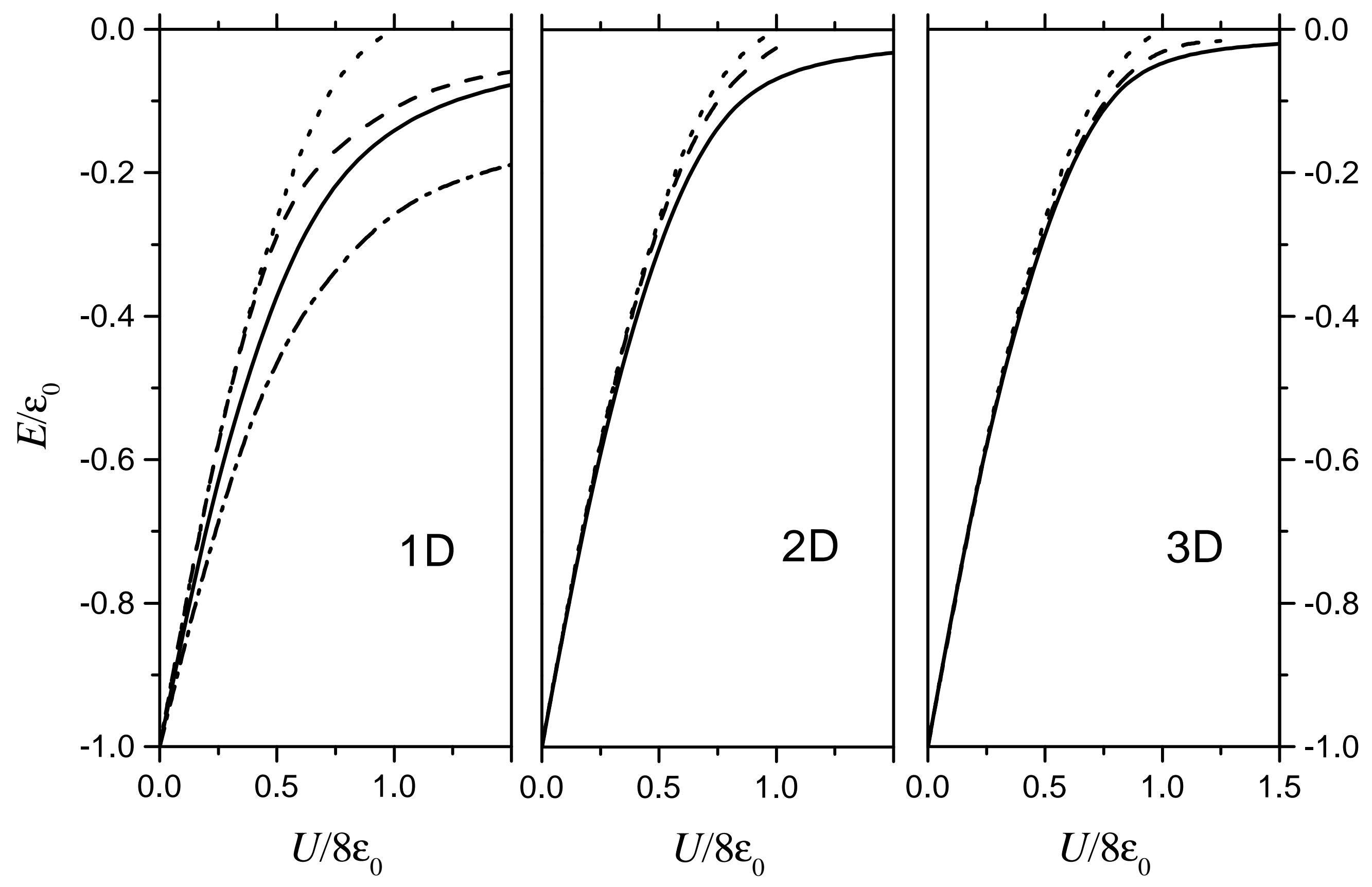




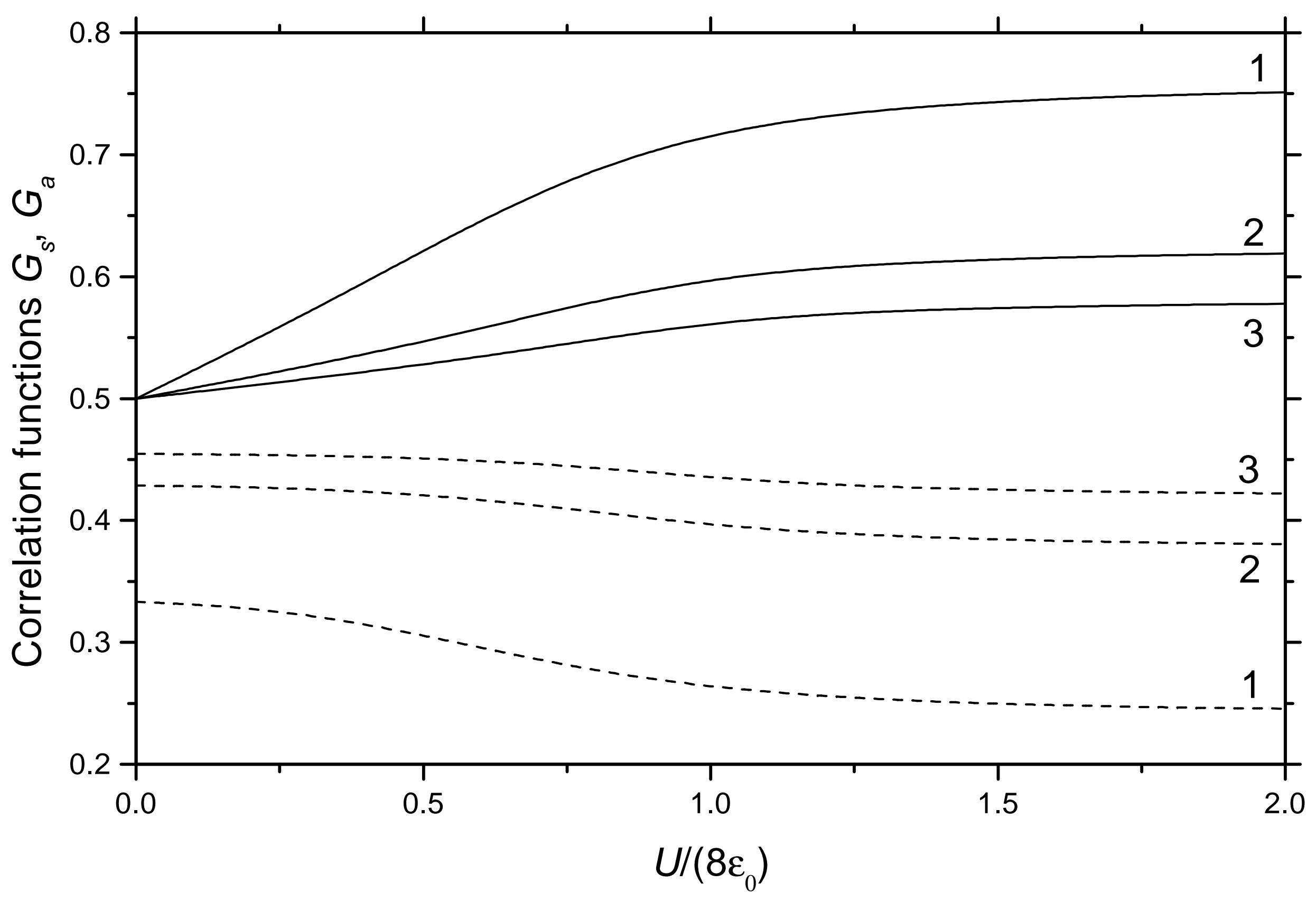

